\title{
Novel Commensurability Effects in Superconducting Films with Antidot Arrays
}

\author{
G. R. Berdiyorov, M. V. Milošević, and F. M. Peeters* \\ Departement Fysica, Universiteit Antwerpen, Groenenborgerlaan 171, B-2020 Antwerpen, Belgium
}

(Received 7 November 2005; published 23 May 2006)

\begin{abstract}
Vortex configurations in superconducting films with regular arrays of antidots (holes) are calculated within the nonlinear Ginzburg-Landau theory. In addition to the well-established matching phenomena, we predict (i) the nucleation of giant-vortex states between the antidots, (ii) the combination of giant- and multivortices at rational matching fields, and (iii) for particular values of the vorticity, symmetry imposed creation of vortex-antivortex configurations.
\end{abstract}

Periodically engineered systems with an energy landscape with many degenerate minima can be found in various areas of physics and they often exhibit mutually common phenomena. Typical examples are the colloidal crystals on periodic substrates or light arrays [1], biomolecular chains formed in an array of obstacles [2], ordering of atoms and molecules adsorbed on corrugated surfaces [3], the electron configurations in arrays of quantum dots (i.e., "quantum cellular automata" [4]), or lattices of superfluid vortices in Bose-Einstein condensates interacting with periodic optical traps [5].

In superconductivity, a similar system has been realized in films with regular arrays of holes (antidots). Namely, direct imaging experiments [6], magnetization, transport, and ac-susceptibility measurements [7] have shown that the flux lines form highly ordered configurations at integer $H_{n}=n \Phi_{0} / S$ and at some fractional $H_{p / q}=\frac{p}{q} \Phi_{0} / S(n, p$, $q$ being integers) "matching" magnetic fields (here $\Phi_{0}=$ $h c / 2 e$ is the flux quantum, and $S$ is the area of the unit cell of the antidot lattice). These commensurability effects between the pinning and the vortex lattice have also technological relevance, as the collective locking of vortices to the pinning sites causes enhanced critical current of the sample.

Following the experimental studies, extensive molecular dynamics simulations (see Ref. [8] and references therein) have been performed within the London limit to study the vortex configurations and their dynamics in a periodic pinning potential. Those vortices were crudely considered as classical point particles and the pinning was simply introduced through a model attractive potential. Since vortices are extended objects which interact in a nontrivial way with the antidot lattice, we here apply the full Ginzburg-Landau (GL) theory, which allows for any vortex rearrangement, their merging, coupling, and any other feature imposed by the geometry and/or pinning strength of the antidot lattice. As will be shown in this Letter, such features lead to novel commensurability phenomena and unanticipated vortex structures.

The superconducting (SC) state in our formalism is described by the Gibbs energy functional, which takes into account not only the intrinsic energy of the sample, but also the energy contribution due to the deformation of the magnetic field lines around the superconductor. The difference between the SC and the normal state Gibbs free energy $(G)$ can be expressed through the integral

$$
\begin{aligned}
\Delta G= & \frac{H_{c}^{2}}{8 \pi} \int\left[-|\psi|^{2}+\frac{1}{2}|\psi|^{4}+\frac{1}{2}|(-i \nabla-\mathbf{A}) \psi|^{2}\right. \\
& \left.+\kappa^{2}\left(\mathbf{h}-\mathbf{H}_{a}\right)^{2}\right] d V,
\end{aligned}
$$

where $\kappa$ denotes the GL parameter, a property of the given material determining the screening of the external magnetic field $\mathbf{H}_{a}$. In Eq. (1), all distances are scaled by the coherence length $\xi$, the vector potential $\mathbf{A}$ by $c h / 2 e \xi$, the magnetic field $\mathbf{h}$ by $H_{c 2}=c \hbar / 2 e \xi^{2}=\kappa \sqrt{2} H_{c}$, and the order parameter $\psi$ by its equilibrium value in the absence of the magnetic field. The minimization of Eq. (1) leads to the well-known GL equations, which we averaged over the SC thickness (being much smaller than the characteristic lengths $\xi$ and $\lambda$ ), mapped on a uniform Cartesian grid (with more than 10 points per $\xi$ ), and solved self-consistently for A and $\psi$ using the iterative procedure from Ref. [9] in combination with the link-variable approach [10]. Our simulation region is a $W_{S} \times W_{S}$ square, mostly with $W_{S}=$ $4 W$ where $W$ is the period of the antidot lattice (i.e., we simulate $4 \times 4$ unit cells; see Fig. 1). The periodicity of the sample is included through the boundary conditions for $\mathbf{A}$ and $\psi$ in the form $\mathbf{A}\left(\mathbf{r}+\ell_{i}\right)=\mathbf{A}(\mathbf{r})+\nabla \zeta_{i}(\mathbf{r})$, and $\psi(\mathbf{r}+$ $\left.\ell_{i}\right)=\psi \exp \left(2 \pi i \zeta_{i}(\mathbf{r}) / \Phi_{0}\right)$, where $\ell_{i=x, y}$ are the lattice vectors, and $\zeta_{i}$ is the gauge potential fulfilling the Landau gauge $\mathbf{A}_{a}=H_{a} x \mathbf{e}_{y}$ for the external vector potential (e.g., $\left.\zeta_{x}=H_{a} W_{S} y, \zeta_{y}=0\right)$. Note that the chosen values of $H_{a}$

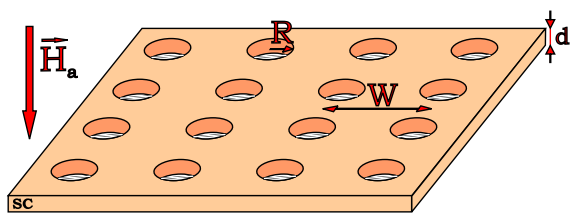

FIG. 1 (color online). Oblique view of the system: the square simulation region containing $4 \times 4$ unit cells. 
must provide the flux quantization per simulation area, as required by the virial theorem [11].

Equilibrium vortex configurations. - We studied the vortex structure of a SC film (with thickness $d$ ) with a square array of circular holes (of radius $R$ ) exposed to a homogeneous magnetic field $H_{a}$ (see Fig. 1). For a given magnetic field the final vortex configuration depends on the geometrical parameters of the antidot lattice. As a representative example, we constructed the equilibrium vortex phase diagram for the forth matching field $\left(H_{a}=H_{4}\right)$, as a function of $W$ and $R$, shown in Fig. 2 for $d=0.1 \xi$ and $\kappa=$ 0.45 . Out of 4 vortices per unit cell, $N_{h}$ vortices are captured by the hole, and the remaining ones sit at interstitial sites. From this phase diagram we notice (1) that the occupation number $\left(N_{h}\right)$ of each hole depends not only on the hole radius $R$ (as discussed in previous works [12]), but also on $W$, i.e., the proximity of the neighboring holes in the lattice. (2) The final configuration of the flux lines is determined not only by their mutual repulsion, but also by the attraction by the antidots and the repulsion by their pinned vortices. Therefore, instead of having an Abrikosov lattice, for close spacing $W(<15 \xi)$, the flux lines form square-shaped lattices regardless of $N_{h}$ (see the insets on the right of Fig. 2). Note that these configurations have been observed experimentally using the Lorentz microscopy in Ref. [6]. (3) With decreasing period $W$, the interstitial vortices become strongly caged between the

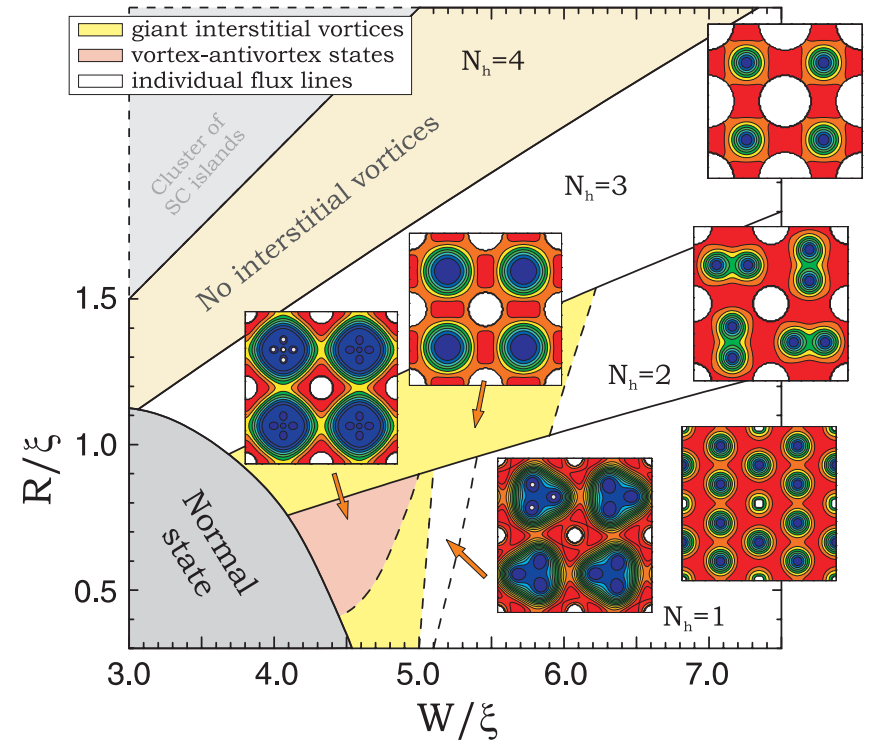

FIG. 2 (color online). The equilibrium vortex lattices at $H_{a}=$ $H_{4}$, as a function of the antidot radius $(R)$ and the period of the antidot lattice $(W)$. The solid lines denote the first order transitions between the states with different antidot occupation number $\left(N_{h}\right)$, and dashed ones depict second order configurational transformations. The insets denote the Cooper-pair density plots [darkest color denotes vortices; white, antidots (blue/red or dark gray/medium gray denotes low/high density)] of the corresponding states (open dots are a guide to the eye indicating the position of the zeros of the order parameter). neighboring antidots, resulting in the disruption of the individual-vortex lattice. The best example is the part of the diagram for $N_{h}=1$, where the caging effect first causes the formation of triangular multivortices (MVs) at interstitials, with chosen orientation that minimizes the energy between the neighboring cells. However, these triangular structures do not follow the imposed square symmetry, and with further decrease of $W$, the confinement causes the formation of the giant-vortex (GV) at each interstitial site (see corresponding area in Fig. 2). Note that in bulk superconductors and nonstructured films the energy of a collection of single vortices has always lower energy than a multiple vortex. The GV state was predicted previously for SC disks [9] and recently detected experimentally [13]. This is the first time that such a state is anticipated for an open geometry, where now the symmetry of the pinning lattice (antidots) forces the interstitial vortices into a giant one. (4) Surprisingly, there is a small area in the phase diagram where a vortex-antivortex (VAV) pair is created, resulting in a configuration with 4 vortices in a square $\mathrm{MV}$, with an antivortex in the center (resulting in five zeros of the order parameter, see the leftmost inset of Fig. 2). These symmetry imposed VAV states are related to the ones found in finite SC polygons [14-16]. As a novelty, we realized VAV states in an open structure, where their fourfold symmetry conforms with the antidot lattice, contrary to the incommensurate triangular vortex arrangement (see insets of Fig. 2). This commensurability in geometry between the antidot lattice and the vortex lattice is a direct consequence of the competing effects between the vortex currents and the screening currents maximal around the antidots (i.e., sample edges). While they are forced to coexist, these currents interfere and compensate each other for small period $W$. Intuitively, lowest resulting currents (and lowest energy state) are achieved for aligned symmetries of antidot and vortex lattices.

In order to verify these findings, and to show they are not peculiar for one chosen field, we repeated our analysis for the rational matching field $H_{a}=H_{9 / 2}$. The ground state $W-R$ diagram is shown in Fig. 3. For sufficient spacing between the antidots, the vortex configuration consists of individual vortices, where a kind of vortex lattice is established by one extra vortex (compared to $H_{a}=H_{4}$ ) being shared between the adjacent cells. Alternatively, every other interstitial site contains this extra vortex (at each site a MV is formed, but with different vorticity). As a consequence, for a dense antidot lattice, the confinement or symmetry does not act equally on adjacent cells-e.g., for $N_{h}=1$, at one site MV with vorticity 4 obeys the imposed symmetry, whereas the neighboring triple vortex is compressed into a giant form [Fig. 3(a)]. The simultaneous presence of a stable giant and a multivortex is unique and has never been predicted before. For $N_{h}=2,3$ vortices at one of the interstitials are not favored by symmetry [Fig. 3(d)], and for small spacing $W$ a VAV configuration 

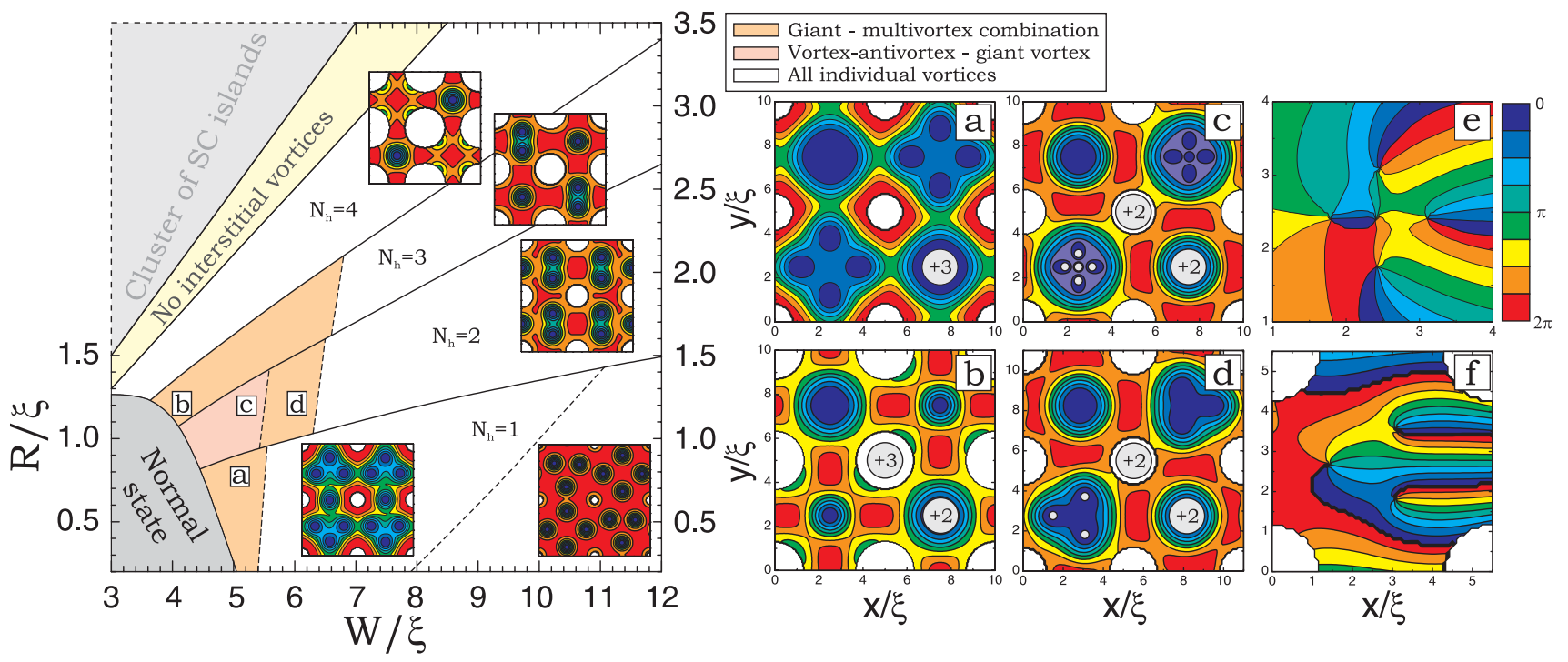

FIG. 3 (color online). The ground-state vortex lattices at $H_{a}=H_{9 / 2}$ fractional matching field (same notation as in Fig. 2 is used). (e)-(f) show the phase of the order parameter (see enclosed scale) of the bottom-left unit cell for the vortex configurations depicted in (c) and (d), respectively.

is induced, in combination with a giant vortex at adjacent site [Fig. 3(c)], illustrating the remarkable variety of possible vortex structures.

Influence of the GL parameter.-It is well known that the GL parameter $\kappa=\lambda / \xi$ (in thin superconductors, $\kappa^{*}=$ $\left.\kappa^{2} \xi / d\right)$ describes the affinity towards type-I or type-II behavior. This GL parameter describes the effective interaction between the vortices. One of the energy terms in this interaction directly depends on $\ln \kappa$, and therefore changes sign when $\kappa$ is decreased. For that reason, although it was shown that VAV configurations in type-II SC polygons are very difficult to be distinguished (VAV spacing $<0.25 \xi$ [15]), Misko et al. [16] argued that VAVs are more distinct and stable in type-I samples, due to the vortex-antivortex repulsion. Motivated by this, we performed the same analysis, and studied the behavior of the vortex structures shown in Figs. 2 and 3 as a function of $\kappa$. We found for decreasing $\kappa$ that the vortex-vortex attraction dominates the phase diagram, and three main features were observed: (i) due to the attraction between the vortices in the holes and the interstitial ones, the threshold hole radius for capturing another vortex lowers (solid lines in Figs. 2 and 3 move to smaller $R$ ); (ii) the giant-vortex state becomes energetically favorable at the interstitials and spreads over the majority of the $W-R$ phase diagram; (iii) due to fact (ii), the VAVs stability region shrinks, and eventually disappears, contrary to the findings in finite SC polygons.

Influence of temperature. - As follows from our formalism [see Eq. (1)], all the sizes in our analysis so far were expressed in units of the coherence length $\xi$. In order to direct the experiment, we will now use conventional units and introduce temperature in the calculation through the $\xi(T)=\xi(0) / \sqrt{1-T / T_{c}}$ dependence.
Figure 4(a) shows the $H_{a}-T$ equilibrium diagram for the VAV state in a $\mathrm{Pb}$ sample $[\xi(0) \approx 40 \mathrm{~nm}, \kappa \approx 1]$ with $W=600 \mathrm{~nm}$ and $R=110 \mathrm{~nm}$. For $H_{a}=H_{4}$ and $T=$ $0.85 T_{c}$ we are located in the middle of the VAV region of Fig. 2. Notice that, e.g., for $H_{a}=H_{5}$ a new reentrant behavior (non-VAV vs VAV states) is found as a function of temperature. For $T \approx T_{c}\left(H_{5}\right)$, since $\xi(T)$ is large, only one vortex can be pinned by each antidot and the remaining 4 are compressed into a giant interstitial vortex. As $T$ decreases, so does $\xi(T)$, vortices gain more space, and the GV may split into a more energetically favorable multivortex. However, further decrease of $T$ makes the holes larger in units of $\xi$ and enables them to capture two vortices each. When left with only 3 vortices per interstitial site, the unit cell imposes the square geometry and the VAV configuration nucleates [analogous to the one
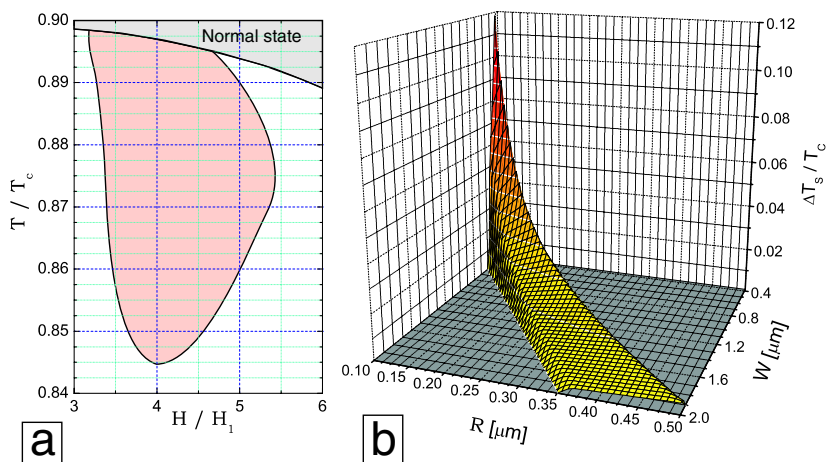

FIG. 4 (color online). (a) The $H_{a}-T$ area of stability of different VAV states for $W=600 \mathrm{~nm}, R=110 \mathrm{~nm}$, and $\kappa=$ 1. (b) $\Delta T$ stability range of the VAV-GV state [see Fig. 3(c)] as a function of the geometrical parameters of the antidot lattice, for $H_{a}=H_{9 / 2}$. 


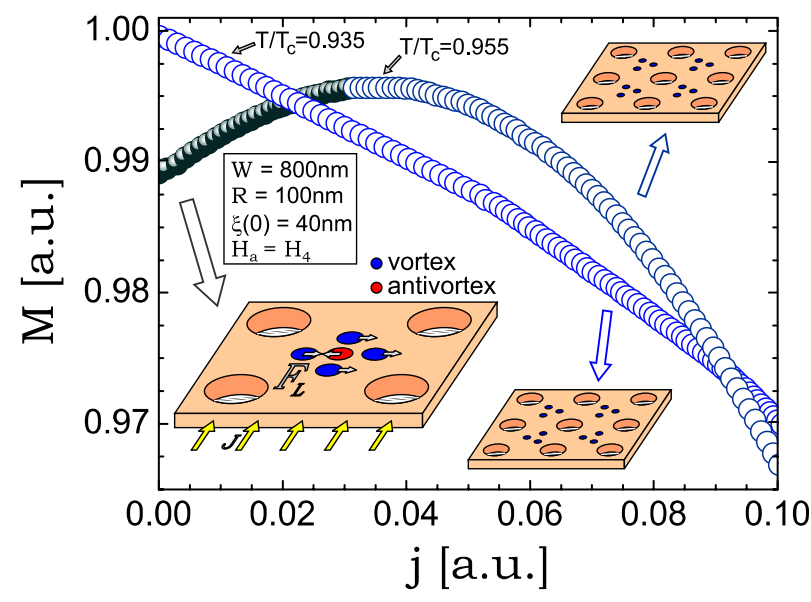

FIG. 5 (color online). Magnetization as a function of the applied current (see illustrations), in the case when VAV pairs are present in the sample (solid dark gray dots) and when they are not (open blue dots).

of Fig. 3(e)]. When the unit cells become too large compared to the vortices, the imposed symmetry influences less the local vortex structure and acts on a vortex lattice as a whole. As a result, a square lattice of individual vortices is formed.

As shown in Fig. 4(a), the VAV-GV state at $H_{a}=H_{9 / 2}$ is stable $\Delta T \approx 5 \% T_{c}$ deep in the SC state. Being important for experimental observation, we calculated $\Delta T$ for VAV state in $\mathrm{Pb}$ films at $H_{a}=H_{9 / 2}$, for different geometrical parameters of the antidot lattice [see Fig. 4(b)]. VAVs were found stable for $W / R \approx 3.81-5.76$, and $\Delta T$ increases with decreasing size of the unit cell and radius of the antidots. The maximal value of $\Delta T / T_{c}=11.3 \%$ is obtained for $W=400 \mathrm{~nm}$ and $R=100 \mathrm{~nm}$. In order to securely stay within the limits of current lithographic techniques, but also within limitations of the GL theory, we did not decrease further $W$ and $R$, but the tendency of even further increase of $\Delta T$ is evident from Fig. 4(b). Note that similar conclusions can be drawn from Ref. [15] for VAV stability in mesoscopic SC squares, but the vortex-antivortex spacing in our case is significantly larger than in the SC polygons [ $>0.75 \xi$, see Fig. 3(e)].

Experimental verification. - The previous temperature stability analysis shows a better prospect for the experimental verification of the predicted VAV states than Ref. [14]. In this respect, we suggest a convenient method, based on the response of the sample to an applied electric current. The idea relies on the Lorentz force, which for an applied dc current acts in the opposite direction on vortices and antivortices, causing their annihilation (see bottom-left inset of Fig. 5). We calculated the magnetization of a perforated $\mathrm{Pb}$ film, as the flux expelled from the superconductor $20 \mathrm{~nm}$ above the sample, over a $1 \mu \mathrm{m} \times 1 \mu \mathrm{m}$ area directly above the chosen interstitial site $(4 \pi M=\langle h\rangle-$ $H_{a}$ ), corresponding to a Hall probe measurement. The results are shown in Fig. 5, for $W=800 \mathrm{~nm}$ and $R=$ $100 \mathrm{~nm}$, at $H_{a}=H_{4}$, for two temperatures $T / T_{c}=$ 0.955 and 0.935 . For qualitative comparison, both magnetization and applied current are expressed in arbitrary units. As one can easily recalculate and compare with Fig. 2, for $T / T_{c}=0.955$ we are in a VAVs equilibrium, whereas for $T / T_{c}=0.935$ the three vortices at interstitial sites form triangular multivortex states (see insets of Figs. 2 and 5). When a small current $j$ is injected in the sample, one expects a monotonous decrease of magnetization on $j$, characteristic for SC films. However, in the VAV state, under the influence of the Lorentz force the vortex and the antivortex slowly approach each other and annihilate, effectively enhancing superconductivity (increasing magnetization). After all VAV pairs disappear, the magnetization curve exhibits a negative slope. At lower temperature, when no VAVs are present, the applied current acts equally on all vortices, and since they become smaller in size with decreasing $T$, the magnetization response linearizesmore importantly, the $M(j)$ curve always has a negative slope. Therefore, the $M(j)$ behavior for small currents can be used as an indication of the presence of VAV states, given by the change of slope from positive to negative. Conventional visualization techniques (Hall probe or Lorentz microscopy) measuring local magnetic fields and scanning tunneling microscopy revealing the local superconducting density (particularly at lower temperatures) are potential alternatives for the observation of the variety of novel vortex states predicted in this Letter.

This work was supported by the Flemish Science Foundation (FWO-Vl), the Belgian Science Policy, the JSPS/ESF-NES program, and the ESF-AQDJJ network.

*Electronic address: francois.peeters@ua.ac.be

[1] M. Brunner et al., Phys. Rev. Lett. 88, 248302 (2002).

[2] J.-L. Vioy, Rev. Mod. Phys. 72, 813 (2000).

[3] P. Zeppenfeld et al., Phys. Rev. Lett. 78, 1504 (1997).

[4] C. S. Lent et al., Nanotechnology 4, 49 (1993).

[5] J. W. Reijnders et al., Phys. Rev. Lett. 93, 060401 (2004).

[6] K. Harada et al., Science 274, 1167 (1996).

[7] M. Baert et al., Phys. Rev. Lett. 74, 3269 (1995); V. Metlushko et al., Phys. Rev. B 60, R12585 (1999); A. V. Silhanek et al., ibid. 67, 064502 (2003).

[8] C. Reichhardt et al., Phys. Rev. B 54, 16108 (1996); 57, 7937 (1998); 64, 014501 (2001); B. Y. Zhu et al., Phys. Rev. B 64, 012504 (2001).

[9] V. A. Schweigert et al., Phys. Rev. Lett. 81, 2783 (1998).

[10] R. Kato et al., Phys. Rev. B 47, 8016 (1993).

[11] M. M. Doria et al., Phys. Rev. B 39, 9573 (1989).

[12] G. S. Mkrtchyan and V. V. Shmidt, Sov. Phys. JETP 34, 195 (1972).

[13] A. Kanda et al., Phys. Rev. Lett. 93, 257002 (2004).

[14] L. F. Chibotaru et al., Nature (London) 408, 833 (2000).

[15] T. Mertelj and V. V. Kabanov, Phys. Rev. B 67, 134527 (2003).

[16] V. R. Misko et al., Phys. Rev. Lett. 90, 147003 (2003). 